\title{
PRACTICAL PROBLEMS
}

\section{Employment and hobbies}

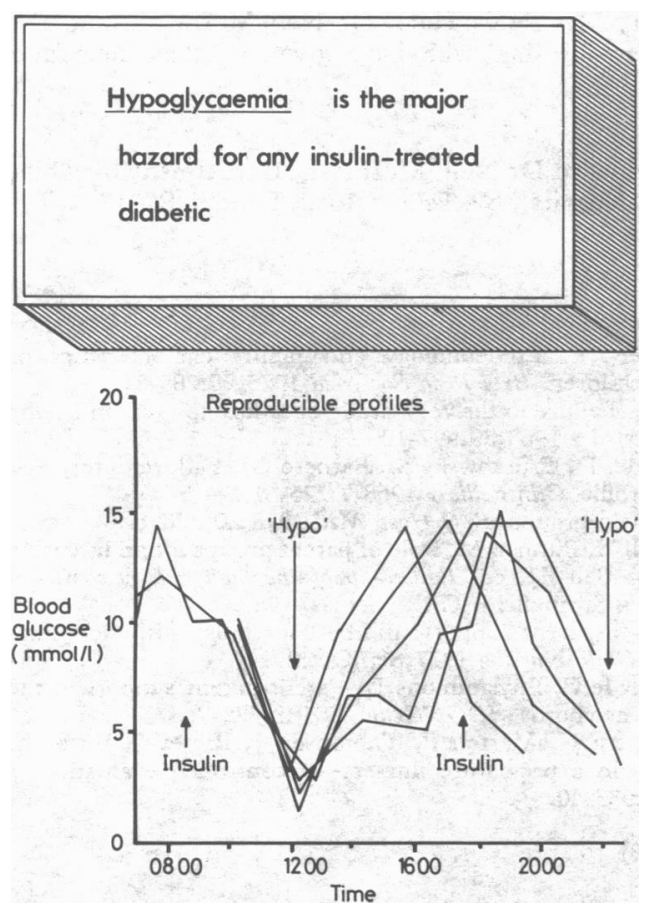

\section{Driving}

"One Sunday morning I think it was, I set off motoring and before lunch began to see double, a sure warning of hypoglycaemia. Not a good thing when you are driving to see two cars or four ditches"

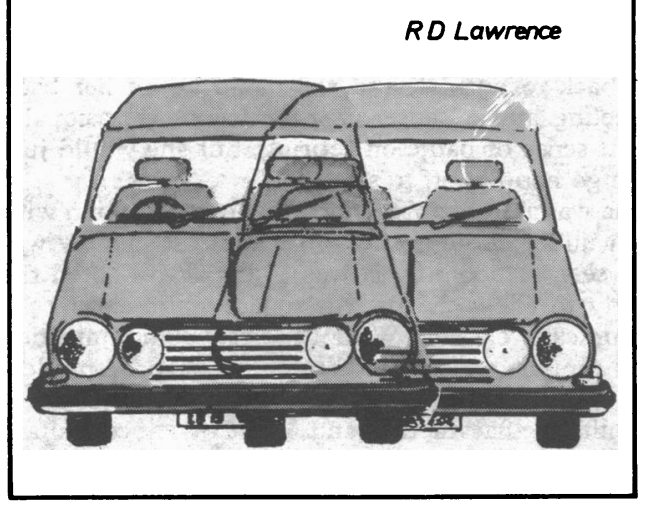

Most of the problems of diabetics in society result from the ever-present possibility of hypoglycaemia in insulin-treated diabetics. Although this hazard is small in many individuals, it is an unacceptable risk in some circumstances. The guiding principle in making the difficult assessments for employment or hobbies is whether the risk of confusion during hypoglycaemia affects the individual only or whether it also places the safety of others at risk.

Individual firms and industries have generally established their own regulations about the suitability of diabetics for particular jobs. If candidates for employment are rejected unreasonably solely on account of diabetes they may appeal. Diabetics are not normally accepted by the armed Forces, the police, the merchant navy, or the fire brigade. Shift work, especially night shift work, should be avoided if possible by those using insulin, but suitable diabetics can certainly make appropriate adjustments and many may successfully cope with such work.

Diabetics treated by diet alone or with oral hypoglycaemic tablets who are otherwise fit should be permitted to undertake any occupation or hobby. Their risk of hypoglycaemia is negligible.

Some diabetics who find difficulty in obtaining work may find it useful to apply for registration as a disabled person. Sometimes this makes it easier for a diabetic to find work. The individual can remove himself from the disabled register at any time.

Diabetics who are otherwise physically fit and not suffering from blackouts are normally allowed to hold ordinary driving licences. The law demands that all new diabetics whether on diet alone, tablets, or insulin should inform the Department of Transport. If applying for a licence for the first time, the appropriate declaration must be made on the application form. It is helpful to indicate whether or not insulin is being used. Driving licences are granted for three years and are reissued (at no extra fee) subject to a satisfactory medical report.

Healthy diabetics treated with diet or tablets are normally allowed to hold both heavy goods vehicle and public service vehicle licences.

Diabetics on insulin treatment may not hold public service vehicle licences and will not normally be permitted to hold heavy goods vehicle licences because of the serious potential consequences of hypoglycaemia, no matter how small the risk may be to any individual diabetic.

All insulin-treated diabetics who drive should always keep a supply of sugar in their cars. They should try not to drive if they are late for a meal, when the danger of hypoglycaemia is great. If they experience warning symptoms of hypoglycaemia, they should stop, switch off the ignition, and preferably leave the car, since they may otherwise be open to a charge of driving under the influence of drugs (insulin). 


\section{Insurance and pensions}

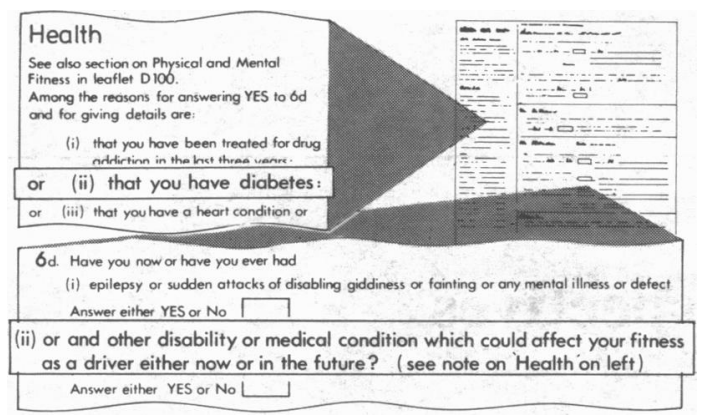

\section{Travel}

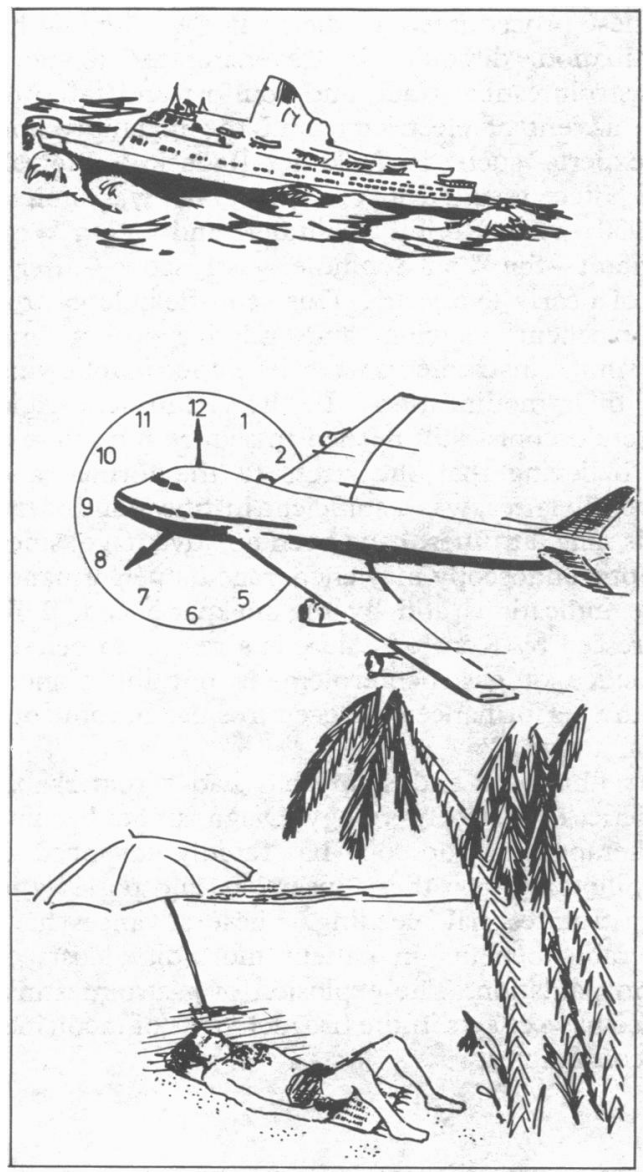

\section{Dental treatment}

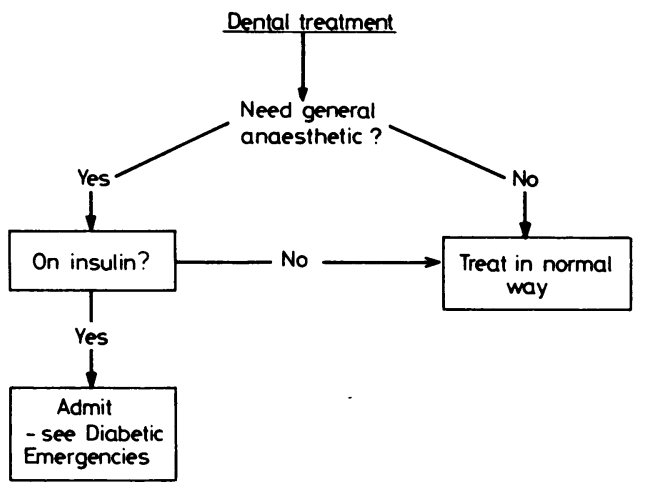

Driving insurance with a normal premium will usually be issued, subject to a satisfactory medical report. Life assurance premiums are often raised by amounts which depend on the result of a medical examination. It is worth looking for the "best buy." Sickness and holiday insurance premiums are often higher than normal. The British Diabetic Association offers helpful advice on insurance.

Small supplementary pensions are available to help diabetics with the increased cost of food for their diets. Patients who are registered blind are eligible for supplementary pensions.

Diabetic control is easily upset by the rigours of travelling. Diabetics should therefore undertake regular urine (or blood) tests and adjust diet and insulin if necessary. Some of the following circumstances present special problems.

Sea sickness-Diabetics may use the same anti-seasickness tablets as non-diabetics: these drugs do not change diabetic control. They all tend to cause some drowsiness, so it is best not to drive while taking them. If vomiting does occur insulin should be continued without fail and the situation dealt with as described in the article on Diabetic Emergencies.

Time changes on long-distance air travel will inevitably make diabetic control difficult for a few days. It is best to change the time of each injection by two or three hours: there should be no difficulty in adjusting to a six-hour change of clock time within 24 hours. If the time between injections is very long a small supplementary injection of soluble insulin (perhaps 4 or 8 units) may be needed if urine tests become very sugary. Conversely, if the time between injections is very short a small temporary decrease of dose may be wise. The urine should be tested every four to six hours during the period of changeover.

Physical activity-More insulin may be needed if the diabetic decides to be much less active when on holiday, or vice versa. Dietary indiscretions may also play havoc with control.

Breakage or loss of equipment-Diabetics should take ample supplies of syringes, insulin, needles, and testing equipment. The equipment in current use and that for emergency use is best kept in separate places. Soluble and isophane insulins may be obtained in most countries, though some of the purified insulins may be less easily found.

Storage of insulin is not generally a problem. In a temperate climate it will keep for some months at room temperature. (Injections sting less if the insulin is not chilled.) Refrigeration, however, would be wise for prolonged visits to tropical climates. It is also recommended if stocks are kept at home for long periods. Insulin should never be deep frozen and should not be left in the luggage hold of an aircraft, where it may freeze.

Vaccination and inoculation are quite suitable for diabetics and should be given for the same indications as for non-diabetics.

Diabetics may receive dental treatment in the normal way and without any special arrangements, except in the case of insulin-treated patients needing a general anaesthetic, when a short admission to hospital is the wisest course.

Dr Peter J Watkins, MD, FRCP, is consultant physician, Diabetic Department, King's College Hospital, London. 